\title{
Influência dos jogos eletrônicos e virtuais no comportamento social dos adolescentes'
}

\author{
Influence of electronic and virtual games in social behavior of the adolescents
}

\author{
Franciely da Silva Cavalli[a], Maria Teresa Ceron Treviso[ ${ }^{[b]}$, Thiago Vendrame ${ }^{[c]}$
}

\footnotetext{
${ }^{\text {[a] }}$ Psicóloga, pós-graduanda em Psicopedagogia na Universidade do Oeste de Santa Catarina (Unoesc), Joaçaba, SC - Brasil, e-mail: francielypsico@yahoo.com.br

${ }^{[b]}$ Docente da graduação e do Programa de Pós-Graduação em Educação, Mestrado em Educação da Universidade do Oeste de Santa Catarina (Unoesc), doutora em Psicologia pelo Instituto de Psicologia da Universidade de São Paulo (USP), São Paulo, SP - Brasil, e-mail: mariateresa.trevisol@unoesc.edu.br

[d Fisioterapeuta pela Universidade do Oeste de Santa Catarina (Unoesc), Joaçaba, SC - Brasil, colaborador da pesquisa, e-mail: thiago.vendrame@yahoo.com.br
}

Recebido: 19/04/2010 Received: 04/19/2010

Aprovado: $13 / 10 / 2011$ Approved: 10/13/2011

\section{Resumo}

O jogo constitui-se uma atividade universal que contribui para o desenvolvimento social, cognitivo e afetivo do ser humano. A percepção dos adolescentes em relação ao significado dos jogos eletrônicos e virtuais em seu cotidiano é a chave para a busca de respostas abordando algumas questões que caracterizem variáveis significativas como definição e importância, discriminação de quantidade de tempo, substituição de relações virtuais por reais, isolamento e possíveis comportamentos agressivos. Nesse sentido, a investigação buscou verificar a influência dos jogos eletrônicos e virtuais no comportamento social e interpessoal dos adolescentes. A parte empírica dessa investigação se deu por meio de um estudo exploratório de natureza quantitativa e qualitativa. A amostra desse estudo foi composta por 203 adolescentes de duas escolas públicas do município de Herval d'Oeste (SC). Como procedimento de coleta de dados, utilizou-se um questionário composto por questões objetivas e subjetivas; e para a análise, utilizou-se o conteúdo das respostas dos sujeitos participantes. Nos dados analisados, evidenciou-se que os adolescentes jogam por diversão, não percebendo componentes cognitivos e físicos que afetam o ato de jogar. A maior parte dos adolescentes, independente de gênero, apresentam preferências por jogos com componentes violentos. Conclui-se que os jogos eletrônicos e virtuais possuem características positivas e negativas de acordo com a percepção e significação do conceito subjetivo de cada indivíduo, tornando-se necessária a atenção ás reações disfuncionais do comportamento derivadas de sinais, sintomas e reações emocionais relacionadas com essa prática.

Palavras-chave: Adolescência. Comportamento social. Videogame. Relações interpessoais.

\section{Abstract}

The game is a universal activity that contributes to social, cognitive and affective development of the human being. The perception of the adolescents regarding the meaning of the electronic and virtual games in his daily life is the key for the answers search boarding some questions

1 Este artigo representa uma faceta de uma investigação intitulada "Play the Game: a influência dos jogos eletrônicos e virtuais no comportamento social e interpessoal dos adolescentes”, realizada no Curso de Pós-graduação em Psicopedagogia pela Universidade do Oeste de Santa Catarina (Unoesc). 
that characterize significant variables as definition and importance, discrimination of quantity of time, substitution of virtual for real relations, isolation and possible aggressive behaviors. In this sense, the investigation looked to check the influence of the electronic and virtual games in the social and interpersonal behavior of the adolescents. The empirical part of this investigation happened through a study exploratory of quantitative and qualitative nature. The sample of this study was composed by 203 adolescents of two public schools of the local authority of Herval d'Oeste (SC, Brazil). How proceeding of collection of data was used a questionnaire composed by objective and subjective questions and for analysis the content of the answers of the subject participants. In the analyzed data it showed up what the adolescents play for amusement, without realizing component cognitive and physical that they affect the act of playing. Most of the adolescents independent of type present preferences for games with violent components. Concluded that the electronic and virtual games have positive and negative characteristics in accordance with the perception and signification of the subjective concept of each individual, becoming necessary pay attention to dysfunctional reactions of the behavior derived from signs, symptoms and emotional reactions related to this practice.

Keywords: Adolescence. Social behavior. Videogames. Interpersonal relations.

\section{Introdução}

Vários fatores contribuem para o desenvolvimento social, cognitivo e afetivo do ser humano, dentre eles o jogo, que constitui uma atividade universal (Huizinga, 2001), que permite a significação e ressignificação de conceitos. Percebe-se que, partindo dessa prática, somos capazes de manifestar alguns conteúdos emocionais que estão diretamente associados às características de personalidade e de relações sociais.

O brincar é uma atividade que apresenta vários significados e assume várias funções como a aprendizagem de regras e a sujeição às ações impulsivas pela via do prazer; ensinar a desejar (Vygotsky, Barreto \& Afeche, 1994); forma mais espontânea de pensamento, fazendo com que o desejável pareça possível de ser obtido (Piaget, 1993); atividade que possibilita a estruturação como sujeito (Freud, 1976). Para as teorias psicogenéticas, o brincar enfatiza a impulsividade, característica do pensamento intuitivo, marcando o início do pensamento lógico, isto é, da heteronomia para autonomia individual no exercício das situações do cotidiano (Alves, 2004).

Como o brincar, os jogos virtuais surgem nos moldes da nossa cultura como sinônimo de prazer, de atualidade, e de inclusão social. 0 jogo, em sua essência, conduz o jogador a uma realidade paralela à sua, ou uma segunda realidade onde ocupa uma postura de fantasia lúdica.
A ascensão da interatividade tem cativado os jovens a explorar o mundo virtual. Inicialmente, trata-se de curiosidade, divertimento e motivação profissional, logo após a interatividade, torna-se uma constante, sendo que consequências comportamentais e físicas podem aparecer de forma gradativa e perceptível na medida em que são utilizados ao extremo. Esses aspectos têm sido explorados por algumas pesquisas que caracterizam os efeitos a curto e longo prazo, indicando em quem provoca danos maiores, as consequências do uso sem limites e a falta de monitoramento dos pais e responsáveis.

Os jogos adentraram nosso contexto com determinação lúdica e com intuito de desenvolver habilidades, no entanto, percebe-se que o foco foi deslocado e o objetivo perdeu-se em meio à disseminação do poder aquisitivo que mantém e motiva a propagação de conteúdos violentos. 0 estudo realizado por Gentile e Anderson (2003) revela que os jogos violentos podem ter consequências negativas, embora não seja provado que os efeitos são idênticos em todas as crianças. Outros estudos apontam que indivíduos que tem por hábito a prática de jogos violentos, possuem a cognição e o comportamento agressivo aumentado (Brady \& Mattews, 2006; Carnagey \& Anderson, 2005; Lynch, Gentile, Olson \& Brederode, 2001). Além disso, a exposição do indivíduo a conteúdos violentos, independente do tempo, possui implicação 
em associações lesivas ou negativas relativas ao desempenho escolar; estes são mais hostis, entram em conflitos e discussões com maior frequência e são mais suscetíveis a agressões físicas (Gentile, Lynch, Linder \& Walsh, 2004; Sharif \& Sargent, 2006).

A prática também influencia ações metabólicas e fisiológicas como frequência cardíaca e pressão arterial, em virtude da intensificação das atividades sedentárias (Wang \& Perry, 2006).

A problemática que constitui o foco deste estudo vem ao encontro da discussão sobre a globalização e evolução tecnológica que a humanidade vem experimentando nos últimos anos. Nesse sentido, buscou-se investigar se há influência dos jogos eletrônicos e virtuais no comportamento social e interpessoal dos adolescentes.

\section{Método}

Trata-se de uma pesquisa descritiva, de cunho exploratório e de abordagem qualitativa e quantitativa. Este estudo contou com uma amostra composta por 203 adolescentes que frequentam duas escolas do Ensino Médio, no período noturno, do município de Herval d'Oeste (SC).

0 início do processo se caracterizou pelo contato com a direção das escolas e a entrega do Termo de Consentimento Livre e Esclarecido previamente aprovado pelo Comitê de Ética em Pesquisa (CEP), da Universidade do Oeste de Santa Catarina sob Resolução n. 016/2009. Esse termo foi entregue aos alunos visando à autorização de seus responsáveis e a proteção ética e sigilo em relação à identificação dos sujeitos de pesquisa.

Para a coleta de dados, foi elaborado um questionário semiestruturado a partir da análise das variáveis constituintes do fenômeno a ser investigado. O conjunto das variáveis explicitadas foi norteador para a elaboração das perguntas que compuseram o mesmo. Os aspectos orientadores para a formulação das perguntas foram:

a) características gerais dos alunos - idade, gênero, turma;

b) representação e significação do conceito de jogo;

c) substituição de relações reais por virtuais;

d) tempo gasto nessas atividades; e) percepção dos adolescentes em relação a essas práticas.

Após a coleta de dados, os questionários foram selecionados e previamente triados. Adotou-se como critério de exclusão o questionário dos alunos que responderam que não jogavam.

Os dados foram analisados de forma a interpretar e explorar a percepção dos adolescentes em relação às práticas dos jogos virtuais. 0 procedimento de análise de dados se baseou no registro do questionário semiestruturado sendo que a informação foi fornecida diretamente pela população pesquisada. Finalmente, a avaliação dos dados deu-se de forma qualitativa visando à análise biopsicossocial do fenômeno investigado.

\section{Resultados}

Participaram do estudo 203 estudantes do Ensino Médio, sendo 62,56\% do gênero feminino e $37,44 \%$ do gênero masculino, com média de idade de 18,15 $\pm 4,35$ anos. Dentre os entrevistados, $79,31 \%$ afirmaram ter jogado algum tipo de jogo eletrônico. Quando observados os gêneros, 72,44\% das mulheres e 90,79\% dos homens afirmaram já terem experimentado essa prática.

O significado atribuído pelos adolescentes aos jogos evidencia que $71,19 \%$ definiram diversão como característica principal da ação de jogar, 15,88\% relacionaram como um modo de interação com os amigos, $4,70 \%$ descreveram como modo de extravasar sentimentos, 4,70\% consideraram parte integrante de seu cotidiano e 3,53\% atribuíram outros significados.

Para a prática dos jogos, o meio mais citado foi o computador, mencionado por $44,95 \%$ dos participantes; o videogame vem em seguida com $27,18 \%$; o celular com $26,48 \%$ e os que utilizam outros meios somam $1,39 \%$.

O tempo destinado diariamente aos jogos, segundo os adolescentes pesquisados, é de menos de uma hora por dia para $64,28 \%$; uma hora por dia para $16,43 \%$; duas a quatro horas diárias para $13,86 \%$; mais de quatro horas ao dia para $5 \%$; e outros períodos de tempo para $1,43 \%$.

Quanto à frequência semanal relatada pelos adolescentes, 47,95\% dizem jogar uma vez na semana; $27,40 \%$ duas ou três vezes por semana; 7,53\% 
jogam quatro ou cinco vezes por semana e $17,12 \%$ afirmam jogar todos os dias.

Ao levantar os aspectos sobre o entendimento dos adolescentes sobre o que consideram jogar demasiadamente: 43,75\% afirmam não importar o tempo que a pessoa joga e sim se o indivíduo abandona atividades para fazê-lo, 22,92\% dizem que é jogar todo dia, $26,25 \%$ afirmam que se torna evidente o jogo excessivo quando a pessoa fica nervosa por não estar jogando, e 2,5\% responderam que é quando se joga mais de uma vez na semana.

Para as situações já vivenciadas: $28,26 \%$ já perderam a hora de refeições para jogar; $20,65 \%$ já deixaram pessoas esperando por atenção após findar a fase ou o jogo; $13,04 \%$ deixaram de ir a passeio com amigos e familiares; $11,41 \%$ preferem jogar a sair de casa; $8,70 \%$ preferem jogar a praticar atividade física; $5,44 \%$ preferem jogar a parar para conversar; $4,89 \%$ já seguiram um modelo virtual transportando-o para a vida real; 4,35\% preferem as relações virtuais às relações reais; e 3,26\% já mudaram de ideia em relação a algum assunto com o conhecimento que adquiriu com os jogos.

Quando questionados sobre as regras, observa-se que estão sendo pouco praticadas conforme respostas dos adolescentes: $77,12 \%$ dizem não haver regras quanto à prática de jogos eletrônicos; $18,30 \%$ afirmam que existem regras, seus pais acompanham e cobram; e 4,58\% afirmam que existem regras, mas como não há cobranças, fica a critério deles seguir a regra ou não.

Quanto à preferência de categorias de jogos, os adolescentes descrevem altos percentuais em esportes $(30,84 \%)$, tiro em $1^{\text {a }}$ e $3^{\text {a }}$ pessoa $(29,34 \%)$, e ação, estratégia e luta $(19,46 \%)$. Os jogos considerados violentos pelos adolescentes caracterizam categorias de tiro em $1^{\underline{a}}$ e $3^{\underline{a}}$ pessoa $(67,43 \%)$, ação, estratégia e luta (28,03\%), esporte $(1,89 \%)$.

Com relação aos sentimentos vivenciados durante a prática, os adolescentes pesquisados afirmaram que sentem irritabilidade $(11,96 \%)$, nervosismo $(8,49 \%)$, sentimento de sucesso $(8,84 \%)$, raiva $(8,32 \%)$, que estão em condições de aprendizagem $(7,80 \%)$, confiança $(7,28 \%)$, poder $(5,37 \%)$, euforia $(5,55 \%)$, sentimentos de companheirismo (5,37\%), alívio $(5,02 \%)$, excitação $(4,51 \%)$, autoestima $(4,33 \%)$, medo $(3,81 \%)$, sentimentos agressivos $(3,47 \%)$, ambição $(3,47 \%)$, frustração $(2,43 \%)$, inclusão no grupo de amigos (1,73\%), fracasso $(1,73 \%)$, isolamento $(0,35 \%)$ e fraqueza $(0,17 \%)$.
Em relação à transferência dos sentimentos, 70,39\% dizem nunca ter manifestado esse comportamento por ter perdido um jogo, porém 15,13\% afirmam já ter desenvolvido esse comportamento uma vez, 12,5\% afirmam já ter desenvolvido algumas vezes, $1,32 \%$ geralmente, e $0,66 \%$ sempre.

\section{Discussĩo}

Com o avanço da tecnologia, os jogos eletrônicos e virtuais invadiram o cotidiano do homem, estando presentes em televisores, celulares, computadores, videogames e demais aparelhos eletrônicos que apresentam facilidades e conforto ao homem. A inserção desses meios na vida social vem tornando-se uma constante quando falamos em relações humanas principalmente com crianças e adolescentes.

0 jogo dá ao jogador a possibilidade de construir representações do cotidiano ou da fantasia em um mundo virtual em que se podem expressar desejos internos desenvolvendo realidades subjetivas que o influenciam fazendo que sua relação com o outro e com as regras morais sejam flexibilizadas. Deste resultam realidades heterogêneas singulares a cada um (Ramos, 2008).

Os adolescentes deste estudo relataram que jogam, em sua maioria, com intuito de diversão e interação com os amigos, o que caracteriza questões típicas do período da adolescência de testagens, descobertas e satisfação egoica, são momentos em que se desencadeiam processos físicos, químicos e psíquicos, os quais são difíceis de compreender e com os quais é difícil lidar. Para exemplificar, selecionamos a resposta do aluno 41 (C.C.C., 18 anos, masculino); segundo ele, é uma "... forma de se divertir, passar o tempo". 0 adolescente busca construir conceitos morais para formar sua identidade social, por isso utiliza recursos de confrontação e simulações, utilizando o jogo como um motivador, estimulando o processo cognitivo (Oliveira, 2007).

Podemos destacar também a função socializadora do jogo que reproduz, mesmo que de maneira lúdica, os padrões, papéis e valores sociais no jogo, favorecendo a apropriação e a experimentação da vida na realidade, como um exercício para a vida em sociedade. Em outro exemplo, identificamos na resposta do aluno 41 (C.C.C., 18 anos, masculino), quando afirma "... No meu celular, videogame, jogo com meus primos". 
Como no estudo de Ferreira e Ribeiro (2000), o computador é o meio mais utilizado por ambos os gêneros $(44,98 \%)$, o que se justifica, já que este proporciona alto nível de interatividade ao jogador, podendo optar por realidades alternativas, a quantidade de jogadores e condições de jogo. As múltiplas opções virtuais e as novas tecnologias que se atualizam diariamente favorecem que outros meios, como aparelho de videogame e celular, sejam extremamente utilizados por jovens com intuito de interatividade lúdica.

Os resultados da investigação realizada se aproximam dos encontrados por Hoff e Wechsler (2002) e Silva, Zapata, Moraes, Doria-Filho e Leone (2006), no que se refere ao tempo de jogo e ao gênero que mais joga. Nesse sentido, evidenciou-se que o gênero masculino joga mais e constantemente se comparado com o gênero feminino.

Os jogos possuem a maioria de seus formatos integrados com personagens do gênero masculino, como relata a organização Children Now (2001), que avaliou personagens de videogame em relação ao gênero. Seus resultados indicam que a possibilidade de interagir com figuras femininas é menor do que com figuras de animais. Isso torna a figura masculina o ideal de poder e agressividade, na medida em que os comportamentos desses personagens giram em torno de agressividade física. Quanto aos personagens femininos, estes são marcados pela agressividade verbal e pela estereotipia do corpo, descrito e exposto como extremamente sexualizado (Alves, 2008).

Os adolescentes deste estudo descreveram características associativas entre a frequência de jogo, sentimentos negativos latentes e atividades sociais em stand-by. Segundo Batista, Quintão e Lima (2008), a frequência inadequada dos jogos pode desenvolver problemas de saúde, emocionais, podendo até configurar-se como vício. 0 jogador passa a necessitar do prazer de jogar, que o deixa ansioso e provoca fugas ou abandono de comportamentos sociais reais, além de insônia, baixo rendimento escolar, falta de paciência para resolver questões que necessitem de elaboração mental mais complexa.

Ao exercer a prática dos jogos, os adolescentes podem perceber ou não a intensidade dos sentimentos, predispondo-se a comportamentos violentos, transportando a agressividade para o contexto social, chegando até a acreditar que essa seja uma prática comum do convívio social. Alguns jogos apresentam conteúdos subliminares imersos em dubiedades escondidas do foco principal de atenção do jogador que propiciam confusão no indivíduo, pois são captadas pelo seu subconsciente memorizando ou expressando valores contrários aos aceitos no cotidiano real (Batista et al., 2008).

A dependência dos jogos eletrônicos e virtuais ocorre à medida que as atividades sociais são substituídas pelas atividades nos jogos eletrônicos e o indivíduo passa a negligenciar atividades de bem comum para interagir somente com situações de cunho eletrônico ou virtual, necessitando deste como método de fuga do real. Para exemplificar essa posição, selecionamos a resposta do aluno 17 (P.C., 24 anos, feminino), que afirma "... o significado dos jogos é a exclusão do mundo real”. Essa prática apresenta-se como problema de saúde pública mundial, considerando que alguns países como Coreia do Sul, Alemanha, Holanda e Estados Unidos já possuem Centros de Tratamento especializados e exclusivos para jovens dependentes de meios eletrônicos (Batista et al., 2008).

Por vezes, um adolescente que gasta horas com os jogos da internet ou videogames poderá perder oportunidades de construir habilidades sociais com pessoas reais ou perder oportunidades de atividade física saudável (Cabral, 2006). Esse dado em relação aos jogos também foi caracterizado pelos adolescentes em segundo plano, com $8,51 \%$ da amostra total, e apresentando porcentagem significativa em relação ao gênero feminino $(12,63 \%)$, o que pode demonstrar a alteração de hábitos saudáveis em decorrência da integração cotidiana da tecnologia.

Atividades como deixar de realizar refeições, de participar de passeios, deixar pessoas esperando e dar prioridade aos jogos, citadas como opção preferencial na vida dos adolescentes, podem prejudicar seu convívio social e familiar. Pensando coletivamente, em sociedade, esses comportamentos geram uma gama de complicações em relação ao diálogo, sistema familiar e social funcional, pois o indivíduo acaba por satisfazer-se mais com relações virtuais do que com as reais, gerando desconforto nas pessoas que fazem parte do seu sistema.

Os sentimentos relatados pelos adolescentes em relação à prática dos jogos apontam uma questão dúbia, pois, mesmo os jovens dizendo que jogam para se divertir, os sentimentos mais citados foram negativos, como irritação e nervosismo. Seria essa 
uma forma de catarse ou a influência dos jogos provoca esse tipo de reação?

As principais críticas aos jogos tratam exatamente dessa questão: os modelos simbólicos estimulam, ensinam ou desinibem comportamentos agressivos? A dessensibilização das situações de violência torna o indivíduo resiliente a ela, banalizando as situações e distorcendo a realidade, criando uma versão paralela e uma percepção paranoide do mundo. Também há a hipótese de que a ativação emocional pode mediar comportamentos entre jogos violentos e agressividade, por aumento da atividade e participação ativa do indivíduo ao vivenciar atividades com conteúdos violentos, o emocional interage com a forma interpretativa das situações, desencadeando irritabilidade e agressividade. Ferreira e Ribeiro (2000) defendem a hipótese da catarse, em que o jogo contribui para liberação da descarga latente da agressividade do indivíduo.

A exposição de crianças e adolescentes a conteúdos violentos, com dubiedade de interpretação ou estereotipagem de conteúdos latentes do cotidiano como forma de estímulo, pode favorecer pensamentos e ações hostis e desarmônicas em relação às aceitas em sociedade (Bushman \& Huesmann, 2006; Gentile et al., 2004; Haninger, Ryan \& Thompson, 2004; Lynch et al., 2001; Olson, 2004). Stateri (2007) remete-se ao critério de que a individualidade do jogador está associada a valores e subjetividade da qual será constituída sua identidade de acordo com sua realidade, tendo plena liberdade de escolha para aceitar regras e definições do jogo, sendo assim responsáveis pela opção e reação decorrentes do ato de jogar. Para Cabral (2006), os jogos constituem espaços de elaboração de conflitos, medos e angústias. Esses jogos ajudam a lidar com emoções e agressividade, prepotência e rebeldia, de um ponto de vista individual e psicológico, com o agravante de banalizar a violência, na medida em que estetizam a morte, a brutalidade e o sangue, tendendo naturalizar aquilo que é essencialmente social, reafirmando e generalizando a violência como um dado da natureza humana.

A violência está propagada nos meios eletrônicos e virtuais com conotação simbólica maior do que a relacionada com a violência física, em razão de imagens mentais que são desenvolvidas no cognitivo do sujeito e alimentadas por questões individuais e sociais. Essas influências podem invocar a memória do pensamento agressivo, despertando desejos de comportamentos violentos (Anderson \& Bushman, 2001), podendo resultar em episódios como o atentado em Columbine, ocorrido em 1999 onde dois jovens, Eric Harris, de 18 anos, e Dylan Klebold, de 17 anos, atiraram em colegas e professores motivados por jogos de videogame. Uma série de tiroteios que aconteceram em Washington levou o caso também de dois jovens de 17 anos a júri; partes afirmavam que os jovens foram treinados para matar enquanto se entretinham com jogos de videogame (Anderson \& Dill, 2000). Segundo Alves (2004), Olson (2004), Ferreira e Ribeiro (2000) e Ramos (2008), a exposição aos conteúdos de violência promove pensamentos e ações violentas que podem desenvolver esse tipo de comportamento.

A falta de regras pela figura de autoridade pode gerar interpretações nas quais os adolescentes acreditam poder ter o controle sobre a prática. Essa atitude também pode ser observada em estudo realizado por Ferreira e Ribeiro (2000); a maioria dos pais permite que os filhos pratiquem jogos tanto tempo quanto quiserem, deixando-os livres para decidir o tempo dispensado, categoria a ser jogada e se essa corresponde à faixa etária adequada às suas necessidades.

Diante dessa situação, a atitude dos pais pode ser compreendida como um descompromisso em relação às escolhas de seus filhos; isso, porém, pode ser explicado pelo fato de que a modernidade trouxe distorções às atitudes parentais, agora marcadas por abnegações de atitudes repreensivas e pela ênfase em atitudes permissivas. Isso denota, inclusive, o entendimento equivocado do Estatuto da Criança e do Adolescente (ECA), que prevê a seguridade em aspectos biopsicossociais.

Outro motivo para esse comportamento é o fato de os pais acreditarem que se o filho está dentro de casa, em frente ao computador ou videogame, está seguro. Infelizmente, esses pais podem estar enganados, pois o perigo pode estar nas associações e leituras que esses adolescentes fazem e a materialização que efetuam dessas informações. É importante estar atento às reações derivadas da prática, pois é possível observar comportamentos disfuncionais como consequências de sentimentos como a frustração.

Segundo Alves (2004) e Ramos (2008), os jogos eletrônicos e virtuais são divididos por temas como ação, estratégia, luta, tiro, RPG, simuladores e 
envolvem em suas entrelinhas uma série de características associadas ao processo cognitivo. Alguns exemplos constituem características das categorias: o RPG (Role Play Game) demanda habilidades simples até complexas avaliações e tomada de decisões; jogos de competição, lutas que definem vencedores e perdedores, produzem sentimentos de inferioridade e de fracasso, superioridade e poder; jogos de tiro ao alvo estimulam a capacidade visual e motora; esporte e corrida são caracterizados por detalhes e precisão; e os simuladores colocam os jogadores em situações vivenciais, proporcionando liberdade de escolha e práticas sequenciais.

Nesse estudo, observou-se que a maioria dos adolescentes mergulha no mundo virtual sem perceber que a maioria dos jogos citados por eles como violentos são os mesmos que, em alguns casos, eles praticam diariamente. Jogos que inicialmente caracterizamos como ação, esporte, ou em sua descrição já indicam a prática de tiros em primeira ou terceira pessoa, também direcionam a vontade do adolescente em satisfazer conteúdos agressivos latentes, podendo transformá-los em comportamento violento real.

Adolescentes acometidos por sinais e sintomas de dependência apresentam comportamentos de frustração e desapontamento quando não jogam ou atingem seus objetivos (Abreu, Karam, Góes \& Spritzer, 2008). Parte desses adolescentes afirma perceber os sintomas físicos e psicológicos como consequência do uso excessivo dos jogos. Batista et al. (2008) indicam que os sintomas do convívio diário com meios eletrônicos e virtuais é percebido quando da descrição de efeitos fisiológicos e psicológicos. Esses trazem como efeito negativo cansaço físico e mental interferindo na produtividade do cotidiano e no desenvolvimento social.

Percebe-se que, como em outros estudos (Alves, 2008; Bushman et al., 2006; Ferreira e Ribeiro, 2000; Lynch et al., 2001; Ramos, 2008), os jogos eletrônicos e virtuais possuem efeitos positivos como reforço para aprendizagem por meio de jogos educacionais, efeitos terapêuticos relativos à catexis dos sentimentos, desenvolvimento de atenção visual, habilidades espaciais, reabilitação e treinamento; no entanto, também apresentam efeitos negativos preocupantes como questões distorcidas de gênero, estímulo ao sedentarismo, estímulo à agressividade e à violência, vício ou dependência, problemas psicológicos e físicos.
É importante ressaltar que os adolescentes desse estudo descreveram alguns dos efeitos negativos, porém não demonstram a capacidade de percepção da situação vivenciada em relação à da situação discutida.

\section{Considerações finais}

Com base nos dados obtidos e mediante os métodos utilizados nesta investigação, é possível perceber as diferenças entre as diversas faces e interpretações dos adolescentes entrevistados, caracterizando a prática dos jogos eletrônicos e virtuais.

Os adolescentes entendem que os jogos funcionam como métodos para extravasarem seus sentimentos e se divertirem. Eles descrevem passar grande quantidade de tempo em frente a máquinas, deixando atividades sociais e familiares em segundo plano, visando à satisfação da conclusão do jogo ou fase naquele momento. Não demonstram perceber que as características que citam como negativas na utilização dos meios eletrônicos são os comportamentos que efetivamente praticam no seu dia-a-dia.

É importante ressaltar a utilização dos meios tecnológicos de forma adequada e com orientação para o desenvolvimento de habilidades como o raciocínio, a lógica, a percepção motora, agilidade de pensamento, atenção, reflexão, estratégias de jogo, planejamento, curiosidade, criatividade, ludicidade, organização, compromisso, respeito e aprimoramento de escolares.

Estudar esses comportamentos, desenvolver trabalhos preventivos, de orientação e de resgate do cotidiano social real, é de impreterível necessidade para que se desenvolvam adolescentes aptos a viver relações saudáveis, mesmo estando em contato efetivo com os meios eletrônicos e virtuais.

\section{Referências}

Abreu, C. N., Karam, R. G., Góes, D. S., \& Spritzer, D. T. (2008). Dependência de Internet e de jogos eletrônicos: Uma revisão. Revista Brasileira de Psiquiatria, 30(2), 156167. doi:10.1590/S1516-44462008000200014.

Alves, L. R. G. (2004). Game over: Jogos eletrônicos e violência. Tese, Doutorado em Educação, Universidade Federal da Bahia, Salvador. 
Alves, L. (2008). Análise das performances em teste de atenção sustentada: Comparação entre jogadores $e$ não-jogadores de videogame. Dissertação, Mestrado em Ciências da Saúde, Universidade Federal de Minas Gerais, Belo Horizonte.

Anderson, C. A., \& Dill, K. E. (2000). Video games and aggressive thoughts, feelings, and behavior in the laboratory and in life. Journal of Personality and Social Psychology, 78(4), 772-790. doi:10.1037/0022-3514.78.4.772.

Anderson, C. A, \& Bushman, B. J. (2001). Effects of violent video games on aggressive behavior, aggressive cognition, aggressive affect, physiological arousal, and prosocial behavior: A meta-analytic review of the scientific literature. Psychological Science, 12(5). doi:10.1111/1467-9280.00366.

Batista, M. L. S., Quintão, P. L., \& Lima, S. M. B. (2008). Influências positivas e negativas dos jogos eletrônicos. V Semanainfo, 2008, Juiz de Fora, MG.

Brady, S., \& Mattews, K. (2006). Effects of media violence on health-related outcomes among young men. Archives of Pediatrics \& Adolescent Medicine, 160(4), 341-347. doi:10.1001/archpedi.160.4.341.

Bushman, B., \& Huesmann, R. (2006). Short-term and long-term effects of violent media on aggression in children and adults. Archives of Pediatrics \& Adolescent Medicine, 160(4), 348-352. doi:10.1001/ archpedi.160.4.348.

Cabral, F. (2006). A captura da subjetividade na guerra dos botões. Sociologia; Ciência \& Vida, 1(2), 32-39.

Carnagey N. L., \& Anderson, C. A. (2005). The effects of reward and punishment in violent video games on aggressive affect, cognition and behavior. Psychological Science, 16(11), 882-889. doi:10.1111/j.1467-9280.2005.01632.x.

Ferreira, P., \& Ribeiro, J. (2000). Os jogos eletrônicos na adolescência: Estudos das diferenças entre os sexos. Dissertação, Mestrado em Psicologia, Universidade Lusófona de Humanidades e Tecnologia, Lisboa.

Freud, S. (1976). Além do princípio do prazer. (Edição standard brasileira das obras psicológicas completas de Sigmund Freud). Rio de Janeiro: Imago Editora.
Gentile, D. A., \& Anderson, C. A. (2003). Violent video games: The newest media violence hazard. In D. Gentile (Ed.). Media Violence and Children (pp. 131152). Westport, CT: Praeger.

Gentile, D., Lynch, P., Linder, J., \& Walsh, D. (2004). The effects of violent video game habits on adolescent hostility, aggressive behaviors, and school performance. Journal of Adolescence, 27, 5-22. doi:10.1016/j. adolescence.2003.10.002.

Haninger, K., Ryan, M., \& Thompson, K. (2004). Violence in teen-rated video games. Medscape General Medicine, 6(1), 1. PMid:15208514. PMCid:1140725.

Hoff, M., \& Wechsler S. A prática de jogos eletrônicos em um grupo de adolescentes. Estudos de Psicologia, 19(2), 2002.

Huizinga, J. (2001). Homo Ludens. São Paulo: Perspectiva.

Lynch, P. J., Gentile, D. A., Olson, A. A., \& Brederode, T. M. V. (2001). The effects of violent video game habits on adolescent aggressive attitudes and behaviors. Paper presented at the Biennial Conference of the Society for Research in Child Development, University of Minnesota Institute of Child Development, Minneapolis, MN.

Oliveira, R. (2007). O adolescente e os jogos eletrônicos: Padrões de uso e motivos. Universidade de Juiz de Fora, Minas Gerais. Recuperado em 17 jan. 2013, de http://www.pospsicologia.ufjf.br/trabalhos/adolescentes_jogos.pdf

Olson, C. (2004). Media violence research and youth violence data: Why do they conflict? Academic Psychiatry, 28, 144-150. doi:10.1176/appi.ap.28.2.144.

Piaget, J. (1983). Psicologia da Inteligência. Rio de Janeiro: Zahar Editores.

Piaget, J. (1993). Seis estudos de psicologia. Rio de Janeiro: Editora Forense Universitária. (Originalmente publicado em 1978).

Ramos, D. K. (2008). Cibernética: Vias do desejo nos jogos eletrônicos. Tese, Doutorado em Educação, Centro de Ciências da Educação da Universidade Federal de Santa Catarina, Florianópolis.

Sharif, I., \& Sargent, J. (2006). Performance Association between television, movie, and video game exposure and school. Pediatrics, 118(4). doi:10.1542/ peds.2005-2854. 
Silva, C., Zapata, A. L., Moraes, A. J. P., Doria-Filho, U., \& Leone, C. (2006). Utilização do computador e de jogos eletrônicos e avaliação da ergonomia com uso do computador em adolescentes de uma escola privada na cidade de São Paulo. Revista Paulista de Pediatria, 24(2), 104-110.

Stateri, J. (2007). 0 videogame como linguagem expressiva. Recuperado em 10 fev. 2009, de http:// www.panaceadesign.com.br/blog/downloads/ vg_como_linguagem_ex
Vygotsky, L. S., Barreto, L. S. M., \& Afeche, S. C. (1994). A formação social da mente: $O$ desenvolvimento dos processos psicológicos superiores. São Paulo: Martins Fontes.

Wang, X., \& Perry, A. C. (2006). Metabolic and physiologic responses to video game play in 7- to 10 -year-old boys. Archives of Pediatrics \& Adolescent Medicine, 160(4), 411-415. doi:10.1001/archpedi.160.4.411. 